\title{
Does Topical Agent Help Fracture Healing? A Pilot Study Using a Herbal Patch
}

\author{
Lung-Fung Tse', Hi-Shan Cheng1, Chi-Yin Tso', Yuk-Wah Hung1, Leung-Kim Hung2, \\ Jing-Zhou Chen'2, Xue-Lin Zhou ${ }^{3,4}$, Chak-Hei Fung ${ }^{3,4}$, Ellie Suet-Yee Pang,4, \\ King-Fai Cheng3,4, Chun-Kwok Wong3,4,5, Ping-Chung Leung2,3,4* \\ ${ }^{1}$ Department of Orthopaedics \& Traumatology, Prince of Wales Hospital, Hong Kong, China \\ ${ }^{2}$ Department of Orthopaedics \& Traumatology, The Chinese University of Hong Kong, Hong Kong, China \\ ${ }^{3}$ Institute of Chinese Medicine, The Chinese University of Hong Kong, Hong Kong, China \\ ${ }^{4}$ State Key Laboratory of Phytochemistry and Plant Resources in West China, The Chinese University of Hong \\ Kong, Hong Kong, China \\ ${ }^{5}$ Department of Chemical Pathology, The Chinese University of Hong Kong, Hong Kong, China \\ Email: ${ }^{*}$ pingcleung@cuhk.edu.hk
}

Received 11 March 2015; accepted 29 April 2015; published 5 May 2015

Copyright (C 2015 by authors and Scientific Research Publishing Inc.

This work is licensed under the Creative Commons Attribution International License (CC BY).

http://creativecommons.org/licenses/by/4.0/

c) (i) 0pen Access

\section{Abstract}

To observe the safety and effectiveness of a topical herbal agent used to promote fracture healing, a herbal patch containing extracts of three herbs, viz., Flos Carthami, Radix Dipsaci, Rhizoma Rhei and Borneolum Syntheticum (an enhancer) was applied on ten subjects with un-displaced fifth metatarsal fractures. Pain scores, foot and ankle function questionnaires and regional swelling were carefully assessed and recorded. Peripheral blood was taken to measure the inflammation cytokines. Assessment checks were performed biweekly to enforce effective patch application and compliance. The results showed that pain improved after two weeks and fracture sites swelling had $20 \%$ reduction in thickness when measured with an ultrasonic tool. Foot and ankle functional scores markedly improved after six weeks. Radiological examinations revealed early perfect fracture unions. The topical herbal patch was effective in promoting fracture healing. It was well tolerated by the fracture patients. Larger randomized controlled trials would be indicated.

\section{Keywords}

Fracture, Traditional Chinese Medicine, Topical Agent, Herbal Treatment

\footnotetext{
"Corresponding author.

How to cite this paper: Tse, L.-F., Cheng, H.-S., Tso, C.-Y., Hung, Y.-W., Hung, L.-K., Chen, J.-Z., Zhou, X.-L., Fung, C.-H., Pang, E.S.-Y., Cheng, K.-F., Wong, C.-K. and Leung, P.-C. (2015) Does Topical Agent Help Fracture Healing? A Pilot Study Using a Herbal Patch. Open Journal of Therapy and Rehabilitation, 3, 35-39. http://dx.doi.org/10.4236/ojtr.2015.32005
} 


\section{Introduction}

Bone fractures are common injuries and the old practice of bone setting has been popular in all communities before the development of modern traumatology [1]. In China, as early as 700BC, principles related to fracture management have been worked out and documented [2]. In Hong Kong today, 14.2\% registered Chinese medicine practitioners (nearly 1000) are bone setters, who serve the injured people with bone setting techniques. They all use topical agents to sooth pain, remove bruises, and reduce swellings related to inflammatory conditions [3]-[5].

This paper is going to discuss about the selection of appropriate medicinal herbs for the study of topical effects. The final selection is based on research platforms pertaining to anti-inflammation, pro-angiogenesis and cellular proliferation. The pilot study is organized according to standard clinical trial requirements and the standard evaluation tools and questionnaires for foot and ankle studies are adopted.

\section{Medicinal Herbs Used in a Novel Topical Agent for Injury Treatment}

Three medicinal herbs in popular use for injury treatment were selected for this pilot study. They were CarthamiFlos (紅花), RheiRhizoma (大黃) and Dipsaci Radix (續斷). In our earlier studies, the three herbs had been shown in the laboratory to be anti-inflammatory, proangiogenic and promoting cellular proliferations. These biological effects were all essential for bone and tissue repair [6]-[8]. The herbs in 1:1:1 proportions were steamed to provide a condensed concoction, freeze-drying of which gave a fine powder preparation, ready to be reconstituted into a paste for topical use in the form of a sticky patch.

A skin sensitivity and irritation test (EpiDerm: EPI-200, MatTek Corporation, Ashland, MA, USA) for the herbal patch was completed to rule out allergens before its clinical applications.

A pilot clinical study was designed to test the effects of the novel herbal patch on fracture healing. We need a fracture site that is superficial and where spontaneous but slow healing is expected. The fifth metatarsal bone fracture is ideal for such purpose: it is a common complication resulting from foot or ankle sprain [9]. In spite of the pain and swelling experienced, these fractures are not displaced and usually only a firm, supportive brace or plaster cast is required to allow careful assisted walking. Fracture healing usually takes more than 8 weeks. The objective of this study was to observe whether the novel herbal patch could promote better fracture healing, help to alleviate pain and swelling, and facilitate rehabilitation.

\section{Methodology}

During the recruitment, the clinical procedures, potential benefits and possible risks of the treatment were explained to the eligible subjects before a written informed consent was signed. The study was designed as a single arm observational pilot trial. Subjects aged 18 or above suffering from undisplaced fifth metatarsal fracture were recruited from the Orthopaedics \& Traumatology clinic at the Prince of Wales Hospital. Before applying the standard orthopedic brace or walking cast, the novel herbal patch was applied over the fifth metatarsal fracture site. The patch was renewed every third day and the treatment lasted a total of 6 weeks. Clinical assessments were given before treatment and every other week until the sixth week.

Clinical assessments included the following:

1) Pain and swelling control;

2) Radiography for the fracture site;

3) Ultrasonography for soft tissue thickness over fracture;

4) Water displacement test to measure foot and ankle swelling; and

5) Using the ScanGoghII imaging system, to generate a 3D image to assess foot swelling [10].

6) Two foot and ankle physical and functional questionaires, viz., Foot and Ankle Ability Measure (FAAM) and American Orthopaedic Foot and Ankle Society ankle-hindfoot scale (AOFAS) were entered to illustrate the functional returns.

The FAAM contained two subscales: activities of daily living (ADL) and sport activities. The scores were transformed to percentage where higher scores indicated a higher level of physical functional [11]. The AOFAS included both subjective and objective data relevant to pain, function and aligniment [12]. Blood samples were taken for the assessment of inflammation cytokines, i.e. TNF-alpha, hs-CRP, IL-1beta, IL-6, IL-8, IL-10 andIL12. During the treatment period, a self administrated diary was used to record the experience of pain by $0-10$ 
numerical rating twice daily in the morning and evening. Compliance as well as concomitant medication were also recorded

\section{Statistical Analysis}

Data analysis was completed according to the intention-to-treat principle. Paired $t$ test was used to compare continuous variables. A two-tailed $p$ value of less than 0.05 was considered significant. All statistical analyses were performed using SPSS version 18.0 (SPSS Inc., Chicago, IL, USA).

\section{Results}

Ten eligible subjects (4 male and 6 female) were recruited to the pilot study. The participants' mean age was 56.5 years (range 26 - 76 years), and the average period after injury was 8.5 days. Radiography confirmed the undisplaced fractures.

Patients experienced significant improvement in morning pain after 2 weeks treatment, $(28 \%$ decrease, $p<$ 0.041); Evening pain took longer to improve, viz., after 4 weeks (41\%decrease, $p<0.026$ ) compared with the baseline. Para-fracture oedema showed a 20\% reduction $(p<0.002)$ with ultrasound measurement in six weeks (Figure 1). An 8\% decrease in 3D foot volume $(p<0.047)$ was also detected after 6 weeks but the water displacement tests failed to reveal differences before and after treatment. Foot and ankle functional assessments showed significant improvements in 6 weeks. In FAAM, ADL improved from $33.3 \pm 23.37$ to $77.9 \pm 21.24$ ( $p<$ $0.001)$ and the Sports activities subscale improved from $13.5 \pm 13.99$ to $48.3 \pm 26.80(p<0.002)$. In AOFAS, the scoring improved from $50.2 \pm 15.22$ to $83.5 \pm 9.11(p<0.001)$ in six weeks.

With regard to the blood analysis, the concentration of most of the inflammatory cytokines in the plasma were either undetectable or too low for analysis with the exception of IL-6, IL-8 and hs-CRP which showed a trend of decline after 6 weeks.

During the treatment period, since pain was well controlled, only three of the ten subjects used occasional additional pain killers (paracetamol) as supplements. The novel herbal patch was well tolerated by all the patients, and no significant adverse reactions, or allergy was experienced. Compliance of patients had been excellent (95\%).

\section{Radiological Study}

Normally undisplaced fractures of the fifth metatarsal heal in 10 - 12 weeks' time and radiological evidence becomes obvious not less than 8 - 10 weeks. In the lower extremities, swelling is expected to persist longer due to gravitational effects, particularly among those allowed to ambulate in spite of the injury. Our volunteers in this pilot study were allowed to ambulate freely while treatment went on.

Radiological studies of our patients revealed that after 6 weeks' topical treatment and bracing, all the fractures healed well. Clinical examinations showed minimal or no tenderness over the fracture site. Toe motions gave

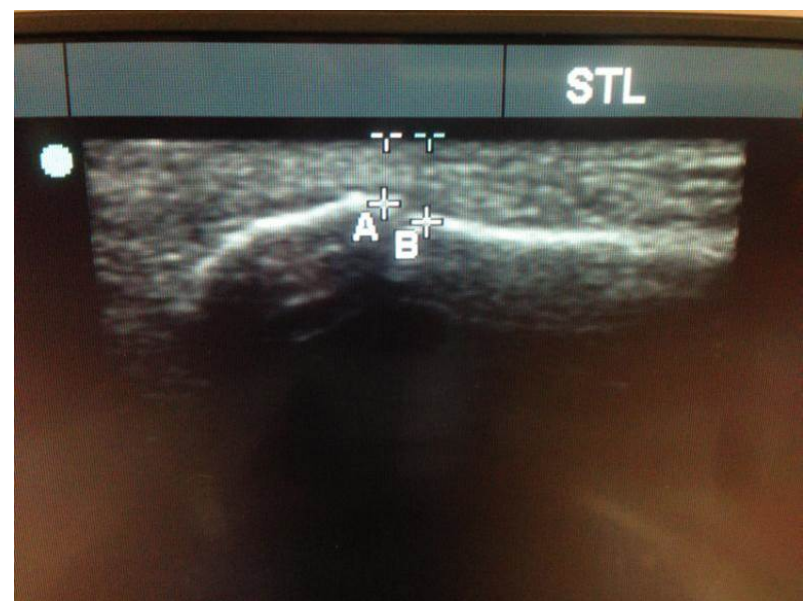

Figure 1. Ultrasonic measurement of para-fracture oedema. 
minimal discomfort, and swelling was mild. Radiologically the bone fractures healed with clear trabecular lines crossing, and good callus formations were obvious (Figure 2). This satisfactory bone healing in 6 weeks' time appeared faster than other situations when no herbal topical agent was used.

\section{Discussion}

One PhD student of our Institute completed a thesis entitled "Pharmacological investigations on the topical herbal treatment effects of bone fracture healing". The herbs she studied showed anti-inflammatory, pro-angiogenic and cellular proliferative effects in laboratory platforms. When the herbs were applied topically over the fracture site of an experimental rabbit, the chemical markers of the herbs used were demonstrated in the rabbit serum, indicating that transcutaneous transport of chemicals derived from the herbs occurred. The fracture healing effects of the topical herbal paste used in the rabbit model was also confirmed, and the treated bone after healing possessed stronger biomechanical properties [13].

The three herbs selected for this pilot study had also been proven anti-inflammatory, pro-angiogenic and cellular proliferation in the laboratory [6]. The results of the pilot study using the herbal patch on the undisplaced fractures of the fifth metatarsal bone were very encouraging: the control of tissue swelling and pain relief in 2 4 weeks' time were very good; the maintenance of ADL and mobilities of the foot and ankle were also good as were shown in the FAAM and AOFAS scores. Moreover, the radiological assessment after 6 weeks showed outstanding bone healing. Adverse events like skin allergy which used to be common with topical agents, were not reported.

\section{Conclusion}

The pilot study using a topical herbal agent to promote fracture healing in the foot showed very positive results. The control of pain and swelling was highly satisfactory and the radiological healing of the bone fractures appeared superior to the usual situation. However, the pilot study involved only 10 patients treated within a short
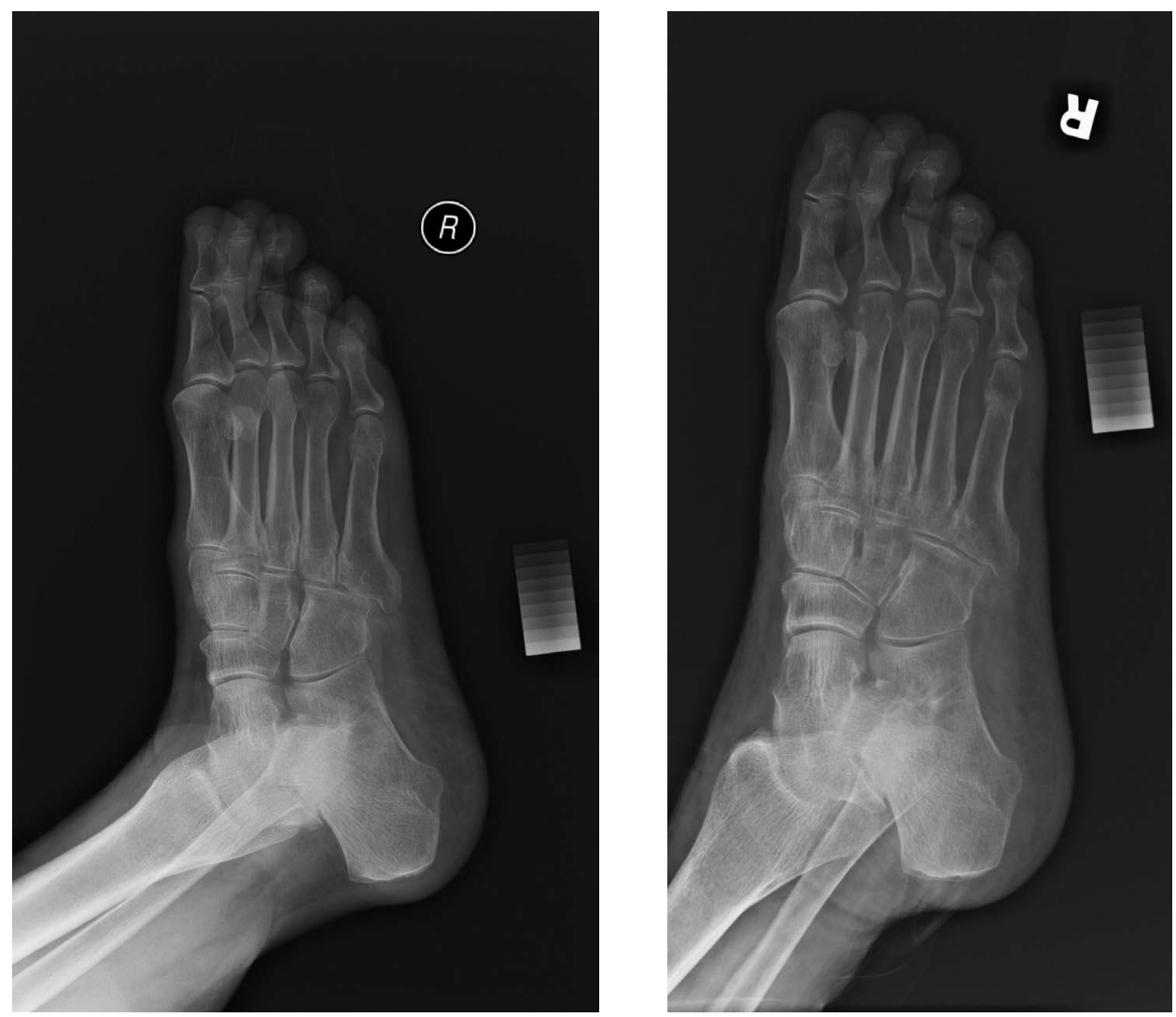

Figure 2. Pre-treatment(left) and post-treatment (right) radiographs showing perfect fracture healing in 6 weeks. 
period of 6 weeks. To further confirm the efficacy of the topical treatment, proper large randomized control trials would be recommended.

\section{Acknowledgements}

This study was financially supported by the Innovation and Technology Commission, the Government of Hong Kong SAR, China (Ref. No. GHX/002/11), and Alberta Technology Limited.

\section{References}

[1] Pettman, E. (2007) A History of Manipulative Therapy. Journal of Manual and Manipulative Therapy, 15, $165-174$. http://dx.doi.org/10.1179/106698107790819873

[2] Wei, Y.Z. (2009) Motor System, Physiology and Blood Circulation. In: Chinese Orthopedic History of Technology, 2nd Edition, Science and Technology Literature Press, Beijing.

[3] Department of Health, HKSAR (2011) Health Manpower Survey Statistics. http://www.dh.gov.hk/english/statistics/statistics_hms/sumcmp11.html

[4] Hong Kong Hospital Authority. http://www.ha.org.hk/visitor/ha_visitor_index.asp?Parent_ID=10042\&Content_ID=10053\&Ver=HTML

[5] Thematic Household Survey Report No. 50, Census and Statistics Department, HKSAR (2013). http://www.statistics.gov.hk/pub/B11302502013XXXXB0100.pdf

[6] Peng, L.H., Ko, C.H., Siu, S.W., Koon, C.M., Yue, G.L., Cheng, W.H., et al. (2010) In Vitro \& in Vivo Assessment of a Herbal Formula Used Topically for Bone Fracture Treatment. Journal of Ethnopharmacology, 131, 282-289. http://dx.doi.org/10.1016/j.jep.2010.06.039

[7] Zhou, X., Siu, W.S., Fung, C.H., Cheng, L., Wong, C.W., Zhang, C., et al. (2014) Pro-Angiogenic Effects of Carthami Flos Whole Extract in Humanmicrovascular Endothelial Cells in Vitro and in Zebrafish in Vivo. Phytomedicine, 21, 12561263. http://dx.doi.org/10.1016/j.phymed.2014.06.010

[8] Leung, P.C., Peng, L.H., Zhao, X. and Hung, L.K. (2010) Rehabilitation Using TopicalAgent—Myth or Reasonable Option? Hong Kong Journal of Occupational Therapy, 20, 3-7. http://dx.doi.org/10.1016/S1569-1861(10)70051-6

[9] Hasselman, C.T., Vogt, M.T., Stone, K.L., Cauley, J.A. and Conti, S.F. (2003) Foot and Ankle Fractures in Elderly White Women: Incidence and Risk Factors. The Journal of Bone and Joint Surgery. American Volume, 85, 820-824.

[10] Vorum Scan Gogh II TM. http://www.vorum.com/english/prosthetics-orthotics/scanners-scangoghII.php.

[11] Kitaoka, H.B., Alexander, I.J., Adelaar, R.S., Nunley, J.A., Myerson, M.S. and Sanders, M. (1994) Clinical Rating Systems for the Ankle-Hindfoot, Midfoot, Hallux and Lesser Toes. Foot Ankle International, 15, 349-353. http://dx.doi.org/10.1177/107110079401500701

[12] Martin, R.L., Irrgang, J.J., Burdett, R.G., Conti, S.F. and Van Swearingen, J.M. (2005) Evidence of Validity for the Foot and Ankle Ability Measure. Foot Ankle International, 26, 968-983.

[13] Peng L.H. (2009) Pharmacological Investigations on the Topical Herbal Treatment Effects of Bone Fracture Healing. Ph.D. Thesis, The Chinese University of Hong Kong., Hong Kong. 